\title{
Lack of Outer Membrane Protein A Enhances the Release of Outer Membrane Vesicles and Survival of Vibrio cholerae and Suppresses Viability of Acanthamoeba castellanii
}

\author{
Soni Priya Valeru, ${ }^{1}$ Salah Shanan, ${ }^{1,2}$ Haifa Alossimi, ${ }^{1}$ Amir Saeed, ${ }^{1}$ \\ Gunnar Sandström, 1 and Hadi Abd $^{1}$ \\ ${ }^{1}$ Division of Clinical Microbiology F 82, Department of Laboratory Medicine, Karolinska Institute, Karolinska University Hospital, \\ Huddinge, 14186 Stockholm, Sweden \\ ${ }^{2}$ Faculty of Medical Laboratory Sciences, University of Medical Sciences and Technology, 11111 Khartoum, Sudan \\ Correspondence should be addressed to Hadi Abd; hadi.abd@ki.se
}

Received 3 December 2013; Revised 10 March 2014; Accepted 10 March 2014; Published 1 April 2014

Academic Editor: Michael McClelland

Copyright @ 2014 Soni Priya Valeru et al. This is an open access article distributed under the Creative Commons Attribution License, which permits unrestricted use, distribution, and reproduction in any medium, provided the original work is properly cited.

\begin{abstract}
Vibrio cholerae, the causative agent of the diarrhoeal disease cholera, survives in aquatic environments. The bacterium has developed a survival strategy to grow and survive inside Acanthamoeba castellanii. It has been shown that $V$. cholerae expresses outer membrane proteins as virulence factors playing a role in the adherence to interacted host cells. This study examined the role of outer membrane protein A (OmpA) and outer membrane vesicles (OMVs) in survival of $V$. cholerae alone and during its interaction with $A$. castellanii. The results showed that an OmpA mutant of $V$. cholerae survived longer than wild-type $V$. cholerae when cultivated alone. Cocultivation with A. castellanii enhanced the survival of both bacterial strains and OmpA protein exhibited no effect on attachment, engulfment, and survival inside the amoebae. However, cocultivation of the OmpA mutant of $V$. cholerae decreased the viability of $A$. castellanii and this bacterial strain released more OMVs than wild-type $V$. cholerae. Surprisingly, treatment of amoeba cells with OMVs isolated from the OmpA mutant significantly decreased viable counts of the amoeba cells. In conclusion, the results might highlight a regulating rule for OmpA in survival of $V$. cholerae and OMVs as a potent virulence factor for this bacterium towards eukaryotes in the environment.
\end{abstract}

\section{Background}

Vibrio cholerae is a curved, rod-shaped, Gram-negative bacterium that causes the severe diarrhoeal disease cholera. Its natural ecosystem includes aquatic environments in endemic locations. Two major virulence factors of $V$. cholerae are cholera toxin (CT) and an intestinal colonization factor known as the toxin coregulated pilus (TCP). Recent studies show that $V$. cholerae has developed a survival strategy to grow and survive inside the free-living amoeba Acanthamoeba castellanii [1-3].

Vibrio cholerae responds to environmental changes by altering the protein composition of its outer membrane (OM). This OM is composed of protein and lipopolysaccharide (LPS) $[4,5]$. However, it has been found that OmpA of
$V$. cholerae shares $47.8 \%$ similarity with that of Escherichia coli. OmpA is a $\beta$-barrel protein in the membrane and is highly conserved among Gram-negative bacteria [6]. It is expressed to very high levels and is tightly regulated at the posttranscriptional level. It can function as an adhesin and invasin, participate in biofilm formation, act as both an immune target and evasin, and serve as a receptor for several bacteriophages $[7,8]$. It has been shown that $E$. coli utilizes OmpA for adhesion to Hela epithelial cells and Caco2 colonic epithelial cells [9].

OMVs are produced by most Gram-negative bacteria, including Vibrio species [10]. The vesicles contain outer membrane proteins, lipopolysaccharides, and phospholipids and, as they are being released from the surface, the vesicles entrap some of the underlying periplasm. They can deliver 
toxins and other virulence factors to the host at relatively high concentrations, without requiring close contact between the bacterial and target human cells, and are believed to represent a key factor in effecting an inflammatory response in the host to bacterial pathogens [11-16].

OMVs are released by Gram-negative bacteria as a novel stress response $[14,17]$, whereas outer membrane proteins (Omp) play a major role in adherence to mucosal membrane in the small intestine and possible protective antigens [14]. The aim of this study was thus to examine the role and influence of OmpA and OMVs in the survival of $V$. cholerae alone and its interaction with $A$. castellanii.

\section{Materials and Methods}

2.1. Microorganisms, Culture Media, and Growth Conditions. The bacterial strains used in this study were wild-type Vibrio cholerae strain A1552 O1 El Tor Inaba [18] and its OmpA mutant by internal in-frame deletion of OmpA gene, kindly provided by Dr. S. N. Wai, University of Umeå. It has been proven that the OmpA mutant bacterium fails to produce OmpA protein, unlike the wild-type strain [7]. Acanthamoeba castellanii was obtained from the American Type Culture Collection (ATCC 30234), Manassas, VA, USA.

$V$. cholerae strains were stored frozen in Luria-Bertani (LB) medium with $15 \%$ glycerol at $-80^{\circ} \mathrm{C}$. Both bacterial strains were grown overnight at $37^{\circ} \mathrm{C}$ on $\mathrm{LB}$ plates and thereafter in LB broth, with shaking to an absorbance ${ }_{600}$ of 0.6 . A castellanii was grown without shaking at $30^{\circ} \mathrm{C}$ to a final concentration of $10^{6} / \mathrm{mL}$ in ATCC medium number 712 .

2.2. Cocultivation. The cocultivation assay was based on a method presented previously [1]. Axenically maintained amoebae were grown at $30^{\circ} \mathrm{C}$ to a final concentration of $2 \times$ $10^{6}$ cell $/ \mathrm{mL}$ in ATCC medium, as described above. Cocultivations of $V$. cholerae with $A$. castellanii were incubated in NUNC tissue culture flasks $\left(75 \mathrm{~cm}^{2}\right)$ purchased from VWR International (Stockholm, Sweden). Each flask contained $50 \mathrm{~mL}$ ATCC medium 712 containing A. castellanii at a concentration of $2 \times 10^{5}$ cell $/ \mathrm{mL}$ and the particular $V$. cholerae species at a concentration of $2 \times 10^{6} \mathrm{cell} / \mathrm{mL}$. Control flasks containing bacteria or amoebae only were prepared in the same way and with the same initial concentration as the coculture flasks. All flasks were prepared in triplicate and incubated at $30^{\circ} \mathrm{C}$. Samples were taken and plated on blood agar plates regularly to study the growth and survival of $V$. cholerae.

2.3. Isolation of Outer Membrane Vesicles and Estimation of Protein Concentration. OMVs were isolated by ultracentrifugation, as described previously [16]. V. cholerae strains were grown in broth culture to late exponential phase. Broth cultures were then centrifuged at $8,000 \mathrm{~g}$ (30 min, $4^{\circ} \mathrm{C}$ ) in a JA-25.50 rotor (Beckman Instruments Inc.). Filtered $(0.22 \mu \mathrm{m}$; Millipore) supernatants were centrifuged at $85,000 \mathrm{~g}\left(2 \mathrm{~h}, 4^{\circ} \mathrm{C}\right)$ in a $70 \mathrm{Ti}$ rotor (Beckman Instruments Inc.) to collect OMVs. Pellets were washed twice with PBS, suspended in PBS to a total volume of $500 \mu \mathrm{L}$, and used as the OMVs preparation. Concentration of total protein in the $\mathrm{OM}$ vesicles was measured spectrophotometrically by the Bradford assay (Bio-Rad).

The effect of outer membrane vesicles on the viability of A. castellanii was examined by incubation of $50 \mu \mathrm{L}$ amoeba cell suspension containing $10^{6}$ cell/mL with $50 \mu \mathrm{L}$ OM vesicle preparation from each bacterial strain or with $50 \mu \mathrm{L}$ BPS for controls. Triplicate experiments were performed and the viability of amoebae was examined after 2 hours by viable count utilizing erythromycin B stain (ATCC).

2.4. Bacterial Adherence. Cocultures of the V. cholerae A1552 and $O m p A$ mutant strains with $A$. castellanii were incubated in $75 \mathrm{~cm}^{2}$ cell culture flasks containing $50 \mathrm{~mL}$ ATCC medium number 712 with an initial concentration of $10^{5}$ cells of $A$. castellanii/mL and $10^{6}$ cells of each $V$. cholerae strain $/ \mathrm{mL}$. The flasks were incubated without shaking at $30^{\circ} \mathrm{C}$ and samples were withdrawn after $1 \mathrm{~h}$ to determine the percentage of bacteria adhering to the amoeba cells. This was done by dividing the number of amoebae with adhered bacteria by the total number of amoebae with and without adhered bacteria and multiplying by 100 .

2.5. Bacterial Uptake, Growth, and Intracellular Survival. The ability of $A$. castellanii to take up $V$. cholerae A1552 strains and the effect of the OmpA mutant on uptake and intracellular growth of the bacterial strains were examined by comparing the interactions of wild-type A1552 and OmpA mutant with the amoebae.

Cocultures of each bacterial strain with $A$. castellanii were incubated in $75-\mathrm{mL}$ cell culture flasks containing $50 \mathrm{~mL}$ ATCC medium number 712 with an initial concentration of $10^{5}$ cells of $A$. castellanii/mL and $10^{6}$ cells of each bacterial strain $/ \mathrm{mL}$. The flasks were incubated without shaking at $30^{\circ} \mathrm{C}$ for $2 \mathrm{~h}$. Each cell suspension was centrifuged for $10 \mathrm{~min}$ at $300 \mathrm{~g}$ in a Labofuge GL centrifuge (VWR International) and washed six times in PBS to remove nonadhered extracellular $V$. cholerae. The pellets were resuspended in $1 \mathrm{~mL}$ PBS and incubated with $500 \mathrm{mg}$ gentamicin/mL for $1 \mathrm{~h}$ at room temperature. The samples were then diluted in $9 \mathrm{~mL}$ PBS and centrifuged for $10 \mathrm{~min}$ at $300 \mathrm{~g}$. Each pellet was resuspended in a volume of $50 \mathrm{~mL}$ ATCC medium in a $75 \mathrm{~cm}^{2}$ culture flask to analyse uptake, intracellular growth, and survival of $V$. cholerae strains. A $1 \mathrm{~mL}$ portion from each flask was centrifuged for $10 \mathrm{~min}$ at $300 \mathrm{~g}$ and each pellet was diluted twofold with $0.1 \%$ sodium deoxycholate to permeabilize the amoeba cells. A series of 10 -fold dilutions of the sample from $10^{1}$ to $10^{10}$ was prepared and spread on blood agar plates. All plates were incubated overnight at $37^{\circ} \mathrm{C}$, and viable counts were performed for the engulfed bacteria. The reculture flasks were incubated without shaking at $30^{\circ} \mathrm{C}$ to investigate the intracellular growth and survival of $V$. cholerae strains using a gentamicin assay and by viable counts for 15 days.

\section{Results}

3.1. Adherence, Uptake, and Intracellular Growth. To estimate adherence of $V$. cholerae wild-type and $O m p A$ mutant strain 


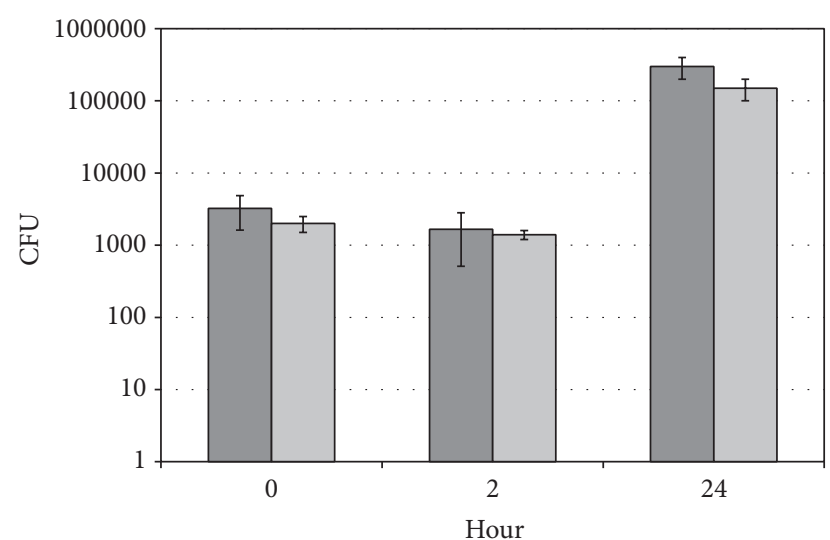

FIgURE 1: Uptake, intracellular growth, and survival of wild-type $V$. cholerae (dark grey bars) and OmpA mutant strains (light grey bars). Zero time is uptake of the bacteria by amoeba cells. Data represent mean \pm SD from three different experiments.

to amoeba cells, the percentage of each bacterial strain adhering to $A$. castellanii was determined and found to be $83.3 \pm 2.1 \%$ and $73.3 \pm 3.5 \%$, respectively. The difference in adherence between the wild-type and $O m p A$ mutant of $V$. cholerae was not statistically significant ( $t$-test, $P=0.29$ ).

To estimate growth and survival of the engulfed bacteria following gentamicin treatment and recultivation, the number of intracellular bacteria was estimated by viable counts. The viable count of uptaken wild-type and OmpA mutant of $V$. cholerae was $3.2 \times 10^{3} \pm 1.6 \times 10^{3} \mathrm{cell} / \mathrm{mL}$ and $4.0 \times 10^{3} \pm$ $5.0 \times 10^{2}$ cell $/ \mathrm{mL}$, respectively (Figure 1 ). The viable counts of intracellular wild-type and $O m p A$ mutant of $V$. cholerae after 2 hours were $1.7 \times 10^{3} \pm 1.2 \times 10^{3} \mathrm{cell} / \mathrm{mL}$ and $1.4 \times 10^{3} \pm$ $2.0 \times 10^{2}$ cell $/ \mathrm{mL}$, respectively, while after 24 hours they were $3.0 \times 10^{5} \pm 1.0 \times 10^{5} \mathrm{cell} / \mathrm{mL}$ and $1.5 \times 10^{5} \pm 5.0 \times 10^{4} \mathrm{cell} / \mathrm{mL}$, respectively (Figure 1). The uptake and intracellular growth of the wild-type and $O m p A$ mutant $V$. cholerae were not significantly different ( $t$-test, $P=0.68)$.

\subsection{Growth and Survival of Wild-Type and OmpA Mutant} $V$. cholerae Cultivated Alone. Viable counts of wild-type and OmpA mutant $V$. cholerae cultivated alone in the absence of A. castellanii showed $10^{6}$-fold increases after 1 day for both. Surprisingly, the wild-type strain survived for only 3 days, while the OmpA mutant of $V$. cholerae survived more than 2 weeks, with a viable count of $1.7 \times 10^{3} \pm 2.1 \times 10^{2} \mathrm{cell} / \mathrm{mL}$ at day 15 (Figure 2). The survival rates of the wild-type and $O m p A$ mutant of $V$. cholerae were significantly different $(t$ test, $P=0.005)$.

\subsection{Growth and Survival of Wild-Type and OmpA Mutant} $V$. Cholerae Cocultivated with A. castellanii. Viable counts of wild-type and OmpA mutant $V$. cholerae cocultivated with A. castellanii showed $10^{3}$-fold increases after 1 day for both strains. Surprisingly, both wild-type and OmpA mutant $V$. cholerae survived more than 2 weeks, but their viable counts were different $\left(2.0 \times 10^{5} \pm 1.0 \times 10^{5} \mathrm{cell} / \mathrm{mL}\right.$ and $4.1 \times 10^{8} \pm$ $2.6 \times 10^{8} \mathrm{cell} / \mathrm{mL}$ at day 15 , resp.) (Figure 3 ).

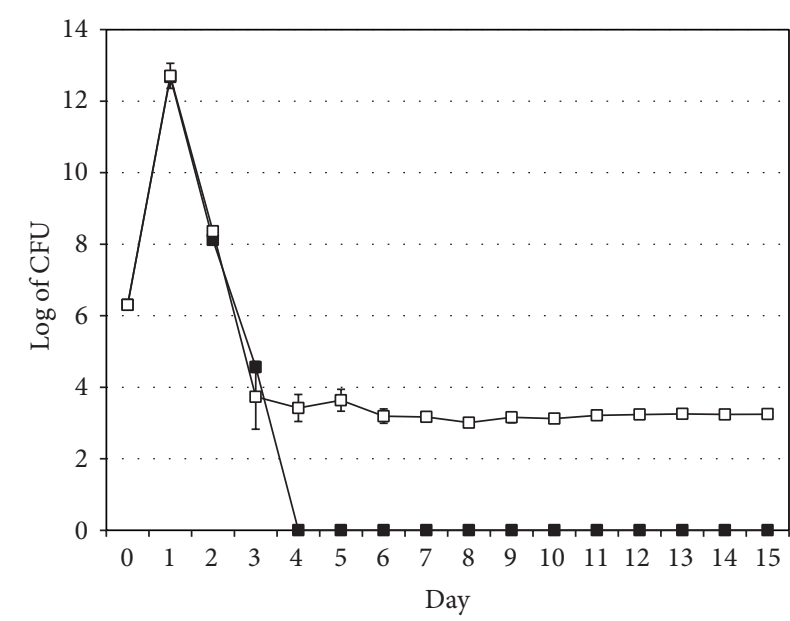

FIgURE 2: Growth and survival of wild-type $V$. cholerae (filled squares) and the $O m p A$ mutant strain (empty squares) cultivated alone. Data represent mean \pm SD from three different experiments.

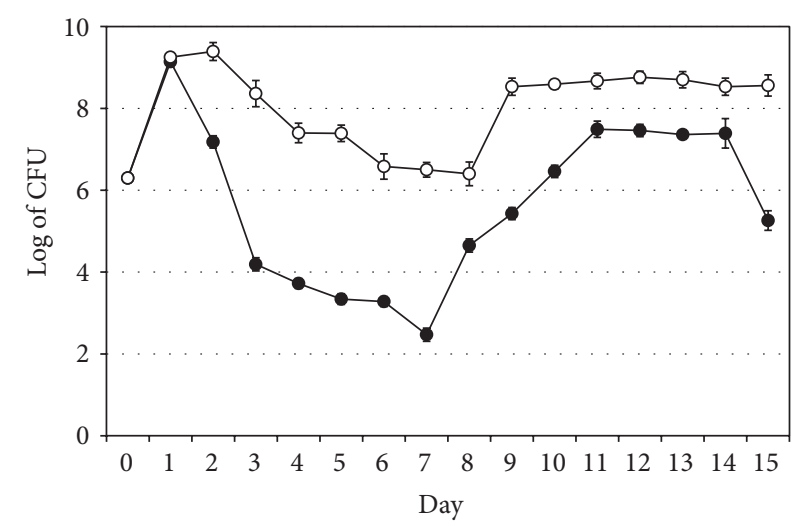

FIGURE 3: Growth and survival of wild-type V. cholerae (filled circles) and the $O m p A$ mutant strain (empty circles) when cocultivated with $A$. castellanii. Data represent mean \pm SD from three different experiments.

The presence of $A$. castellanii enhanced survival and growth of both wild-type and $O m p A$ mutant stains of $V$. cholerae (Figure 3) compared with that in the absence of $A$. castellanii (Figure 2). Growth of the cocultivated wild-type and $O m p A$ mutant strains of $V$. cholerae differed significantly ( $t$-test, $P=0.0004$ ), with growth of the OmpA mutant being higher than that of the wild-type strain (Figure 3 ).

3.4. Growth and Survival of A. castellanii Alone or Cocultivated with Wild-Type and OmpA Mutant Strains of V. cholerae. The number of viable $A$. castellanii alone and cocultivated with wild-type $V$. cholerae increased from $2.0 \times 10^{5} \pm 0.0 \mathrm{cell} / \mathrm{mL}$ to $1.8 \times 10^{6} \pm 4.2 \times 10^{5}$ and $9.3 \times 10^{5} \pm 1.8 \times 10^{5} \mathrm{cell} / \mathrm{mL}$, respectively, after 15 days. In contrast, the number of viable $A$. castellanii cocultivated with the $O m p A$ mutant $V$. cholerae decreased from $2.0 \times 10^{5} \pm 0.0$ cell $/ \mathrm{mL}$ to $1.3 \times 10^{4} \pm 1.0 \times$ $10^{3}$ cell/mL after 15 days (Figure 4 ). 


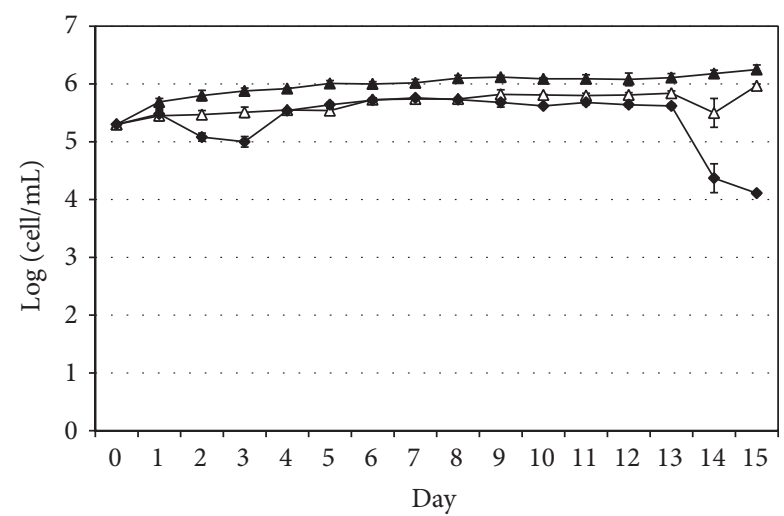

FIgURE 4: Growth and survival of $A$. castellanii cultivated alone (filled triangles), with wild-type $V$. cholerae (empty triangles) and with the OmpA mutant strain of $V$. cholerae (filled diamonds). Data represent mean \pm SD from three different experiments.

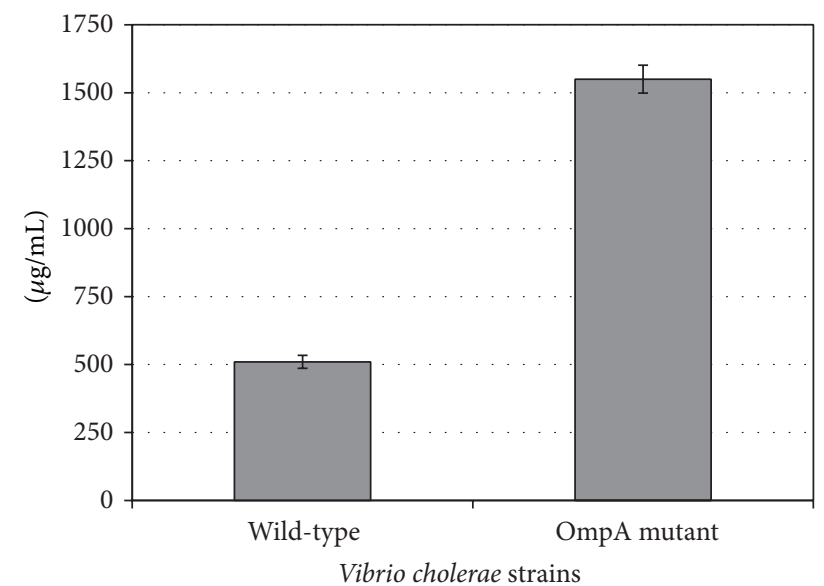

FIGURE 5: Production of outer membrane vesicles by $V$. cholerae wild-type and $O m p A$ mutant strains. Data represent mean \pm SD from three different measurements.

Cocultivation of the amoebae with $V$. cholerae strains showed that the growth rate of $A$. castellanii alone and in the presence of wild-type or OmpA mutant $V$. cholerae was significantly different ( $t$-test, $P=0.00020$ and $P=0.00024$, resp.). However, the difference between the growth rate of $A$. castellanii cocultivated with wild-type or $\operatorname{OmpA}$ mutant of $V$. cholerae was less significant $(P=0.04)$.

3.5. Production of Outer Membrane Vesicles by $V$. cholerae Strains and Effect of OMVs on A. castellanii Viability. OMVs were isolated from the wild-type and $O m p A$ mutant strains as described in Section 2. The amount of vesicles released from the two strains was compared by measuring protein concentration, which was found to be $510 \pm 24 \mu \mathrm{g} / \mathrm{mL}$ for the wild-type and $1550 \pm 51 \mu \mathrm{g} / \mathrm{mL}$ for the OmpA mutant strain (Figure 5). This difference in protein concentration was statistically significant ( $t$-test, $P=0.0001$ ).

Treatment of the amoebae with bacterial vesicles showed that the OMVs lowered the viability of the amoeba cells after 2 hours of incubation. Thus the viable count of $A$. castellanii

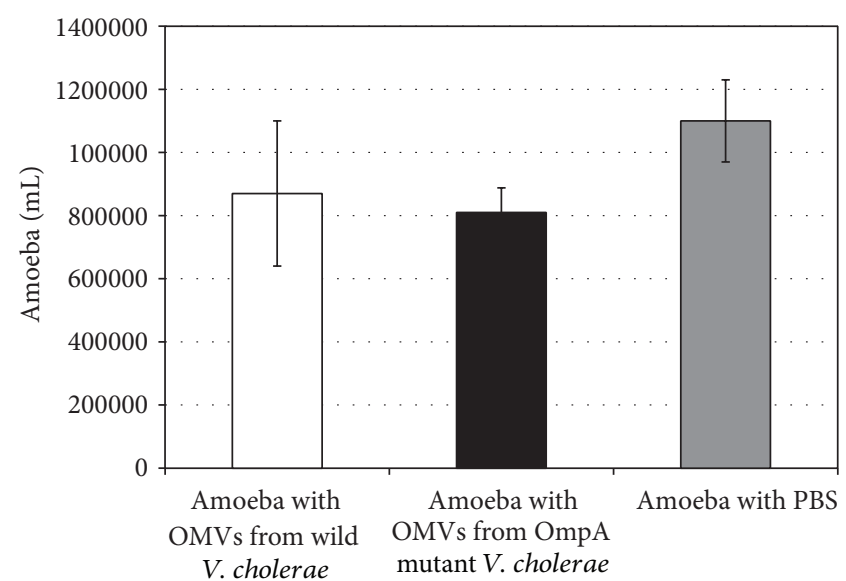

FIGURE 6: Effect of outer membrane vesicles (OMVs) from wild-type and $O m p A$ mutant strains of $V$. cholerae on viability of $A$. castellanii. Data represent mean \pm SD from three different measurements.

incubated with OMVs from wild-type $V$. cholerae, OMVs from the OmpA mutant strain, and PBS was $8.7 \times 10^{5} \pm 2.3 \times$ $10^{5}, 8.1 \times 10^{5} \pm 7.8 \times 10^{4} \mathrm{cell} / \mathrm{mL}$, and $1.1 \times 10^{6} \pm 1.3 \times$ $10^{5}$ cell $/ \mathrm{mL}$, which represent $79 \%, 74 \%$, and $100 \%$ viability, respectively (Figure 6). The viable count decreased significantly compared with the PBS treatment for the amoebae treated with OMVs from OmpA mutant $V$. cholerae ( $t$-test, $P=0.02$ ), but the decrease was less significant for the amoebae treated with OMVs from wild-type $V$. cholerae $(t$ test, $P=0.05)$. However, this difference in amoeba viability might be due to the ability of the OmpA mutant of $V$. cholerae to produce more OMVs, as demonstrated here and by Song et al. [7].

\section{Discussion}

$V$. cholerae utilizes several survival strategies in aquatic environments, such as biofilm formation, switching from smooth to rugose colony morphotypes, and association with free-living amoebae [19]. Studies have shown that $V$. cholerae has enhanced growth in association with A. castellanii [13 ] and both microorganisms have been detected in the same water samples from cholera endemic areas [20]. This study investigated the role of OmpA protein and the outer membrane vesicles released by $V$. cholerae in survival and interaction of the bacterium with the eukaryotic host $A$. castellanii.

The results demonstrated that, in the absence of $A$. castellanii, the OmpA mutant $V$. cholerae survived for much longer ( $>15$ days) than wild-type $V$. cholerae ( 3 days). In this context, loss of all CFU from wild-type $V$. cholerae cultivated in this rich medium in the absence of amoebae was observed not only in this paper but also previously for other bacteria such as Francisella tularensis [21], Shigella dysenteriae, and S. sonnei [22].

Francisella and Shigella species are facultative intracellular bacteria multiplying inside amoeba cells and remaining cultivable during the experiment time compared to the 
bacteria in absence of the amoebae that became noncultivable. Moreover, interaction of the extracellular bacterium Pseudomonas aeruginosa with $A$. castellanii showed that growth and survival of $P$. aeruginosa were the same during the experiment time whether the amoebae were present or absent [23]. However, V. cholerae O1, V. cholerae O139, and $V$. mimicus lost all CFU from the wild-types cultivated in absence of the amoebae from day 4 of cultivation [22, 2426 and it was proven that $V$. cholerae died and did not enter the viable but nonculturable (VBNC) state after the loss of all CFU from the wild type [25]. The question is how the OmpA mutant of $V$. cholerae survived longer than the wildtype strain.

In the present study, it was found that the OmpA mutant of $V$. cholerae produced significantly more OMVs than the wild-type strain, confirming previous findings by Song et al. [7] that the lack of OmpA protein leads to production of more OMVs. An interesting observation in the present study was that significant production of OMVs might have enriched the cultivation medium and supported longer survival of the mutant strain compared with the wild-type V. cholerae.

To investigate the effect of OMVs on the amoebae, $A$. castellanii cells were incubated in a suspension of OMVs isolated from each $V$. cholerae strain. The viable counts demonstrated a decreased viability of $A$. castellanii in both cases; this might indicate a virulence role of the OMVs towards the amoebae, in agreement with other studies [7, 27].

Interaction of $V$. cholerae strains with $A$. castellanii involves attachment of bacteria to the amoeba cells, engulfment, intracellular growth, and survival inside the amoebae. The engulfment, intracellular growth, and intracellular survival of the wild-type and $O m p A$ mutant $V$. cholerae were not significantly different. In this context, Abd et al. [1] found that the capsule and LPS O-side chain did not affect engulfment, intracellular growth, and intracellular survival of $V$. cholerae O139 when interacted with A. castellanii.

The results also showed that the presence of $A$. castellanii enhanced survival of both wild-type and $O m p A$ mutant strains of $V$. cholerae. This was in agreement with previous findings that interaction of $A$. castellanii with wild-type $V$. cholerae O139, the capsule mutant strain, and the capsule/LPS double mutant strain enhanced survival of all these bacterial strains [1]. Moreover, in spite of the fact that $V$. cholerae $\mathrm{O} 1$ El Tor possesses a mannose-sensitive haemagglutinin fimbria and $V$. cholerae $\mathrm{O} 1$ classical does not, they have enhanced survival and their intracellular growth in A. castellanii is not significantly different [2]. All these facts may indicate that the intracellular behaviour of $V$. cholerae is a new survival strategy $[1,2]$.

In this study, the presence of the OmpA mutant $V$. cholerae decreased viability of $A$. castellanii significantly more than the wild-type $V$. cholerae did; this might be due to overproduction of OMVs by the mutant strain. The OMVs of $V$. cholerae have been suggested to promote the delivery of virulence factors to bacterial or eukaryotic cells [27]. However, our results showed that OMVs decreased viability of the amoebae, which might indicate that vesicles are a virulence factor.

It has been observed previously that OmpA level is inversely correlated with the amount of OMVs and that the
sRNA of $V$. cholerae, which is called vibrio regulatory RNA of OmpA (VrrA), increases OMVs production at a rate comparable to the loss of OmpA, since VrrA positively regulates OMVs release through downregulation of OmpA protein [7]. However, inactivation of VrrA resulted in increased colonization of $V$. cholerae in an infant mouse colonization assay. Thus, OmpA protein is important for the colonization of $V$. cholerae, and VrrA RNA may be considered a regulator that modulates the virulence of $V$. cholerae [7].

OMVs formation has been suggested to be linked to turgor pressure of the cell envelope during bacterial growth [28]. Gram-negative bacteria have developed many strategies to enable active virulence factors to gain access to the extracellular environment, typically the tissues or bloodstream of the host organism [29].

Vesicles are the means by which bacteria interact with prokaryotic and eukaryotic cells in their environment. Biochemical analysis and functional characterization of pathogen-derived outer membrane vesicles have demonstrated that this secretory pathway has been taken by pathogens for the transport of active virulence factors into host cells [14]. However, the ability of OMVs to fuse with bacterial membranes and of host cells to deliver content into the cytosol means that these vesicles may be described as bacterial "bombs" for directed intercellular transport of particular bacterial virulence factors into host cells and tissues [14, 30-32]. Further investigations are needed to learn more about the function of the vesicles and their interaction with host cells.

Finely, the results showed that when both strains were cultivated alone, the OmpA mutant of $V$. cholerae expressed more OMVs and survived for longer than the wild-type. Moreover, the amount of OMVs isolated from the OmpA mutant strain was sufficiently high to decrease viability of amoebae. Cocultivation with $A$. castellanii enhanced survival of both wild-type and OmpA mutant strains of $V$. cholerae.

In conclusion, OmpA might have a regulating role in survival of $V$. cholerae since it suppressed its survival while the lack of OmpA enhanced release of OMVs. The OMVs might act as a virulence factor when they supported a long survival of the bacterium and decreased viability of the interacted amoebae. $V$. cholerae might be adapted to survive better in association with eukaryotes.

\section{Conflict of Interests}

The authors declare that there is no conflict of interests regarding the publication of this paper.

\section{Acknowledgment}

The authors gratefully acknowledge the Swedish Civil Contingencies Agency (MSB) for supporting this project.

\section{References}

[1] H. Abd, A. Saeed, A. Weintraub, and G. Sandström, "Vibrio cholerae O139 requires neither capsule nor LPS O side chain 
to grow inside Acanthamoeba castellanii," Journal of Medical Microbiology, vol. 58, no. 1, pp. 125-131, 2009.

[2] H. Abd, A. Saeed, A. Weintraub, G. B. Nair, and G. Sandström, "Vibrio cholerae O1 strains are facultative intracellular bacteria, able to survive and multiply symbiotically inside the aquatic free-living amoeba Acanthamoeba castellanii," FEMS Microbiology Ecology, vol. 60, no. 1, pp. 33-39, 2007.

[3] G. Sandström, A. Saeed, and H. Abd, "Acanthamoeba-bacteria: a model to study host interaction with human pathogens," Current Drug Targets, vol. 12, no. 7, pp. 936-941, 2011.

[4] S. Kabir and P. Mann, "Immunological properties of the cell envelope components of Vibrio cholerae," Journal of General Microbiology, vol. 119, no. 2, pp. 517-525, 1980.

[5] J. T. Kelley and C. D. Parker, "Identification and preliminary characterization of Vibrio cholerae outer membrane proteins," Journal of Bacteriology, vol. 145, no. 2, pp. 1018-1024, 1981.

[6] A. H. Delcour, "Structure and function of pore-forming $\beta$ barrels from bacteria," Journal of Molecular Microbiology and Biotechnology, vol. 4, no. 1, pp. 1-10, 2002.

[7] T. Song, F. Mika, B. Lindmark et al., "A new Vibrio cholerae sRNA modulates colonization and affects release of outer membrane vesicles," Molecular Microbiology, vol. 70, no. 1, pp. 100-111, 2008.

[8] E. Sugawara and H. Nikaido, "Pore-forming activity of OmpA protein of Escherichia coli," Journal of Biological Chemistry, vol. 267, no. 4, pp. 2507-2511, 1992.

[9] A. G. Torrest and J. B. Kaper, "Multiple elements controlling adherence of enterohemorrhagic Escherichia coli O157:H7 to HeLa cells," Infection and Immunity, vol. 71, no. 9, pp. 49854995, 2003.

[10] K. Kondo, A. Takade, and K. Amako, "Release of the outer membrane vesicles from Vibrio cholerae and Vibrio parahaemolyticus," Microbiology and Immunology, vol. 37, no. 2, pp. 149-152, 1993.

[11] C. Balsalobre, J. M. Silván, S. Berglund, Y. Mizunoe, B. E. Uhlin, and S. N. Wai, "Release of the type I secreted $\alpha$-haemolysin via outer membrane vesicles from Escherichia coli," Molecular Microbiology, vol. 59, no. 1, pp. 99-112, 2006.

[12] T. N. Ellis and M. J. Kuehn, "Virulence and immunomodulatory roles of bacterial outer membrane vesicles," Microbiology and Molecular Biology Reviews, vol. 74, no. 1, pp. 81-94, 2010.

[13] J. C. Kouokam, S. N. Wai, M. Fällman, U. Dobrindt, J. Hacker, and B. E. Uhlin, "Active cytotoxic necrotizing factor 1 associated with outer membrane vesicles from uropathogenic Escherichia coli," Infection and Immunity, vol. 74, no. 4, pp. 2022-2030, 2006.

[14] M. J. Kuehn and N. C. Kesty, "Bacterial outer membrane vesicles and the host-pathogen interaction," Genes and Development, vol. 19, no. 22, pp. 2645-2655, 2005.

[15] A. Kulp and M. J. Kuehn, "Biological functions and biogenesis of secreted bacterial outer membrane vesicles," Annual Review of Microbiology, vol. 64, pp. 163-184, 2010.

[16] S. N. Wai, B. Lindmark, T. Söderblom et al., "Vesicle-mediated export and assembly of pore-forming oligomers of the enterobacterial ClyA cytotoxin," Cell, vol. 115, no. 1, pp. 25-35, 2003.

[17] A. J. McBroom and M. J. Kuehn, "Release of outer membrane vesicles by gram-negative bacteria is a novel envelope stress response," Molecular Microbiology, vol. 63, no. 2, pp. 545-558, 2007.

[18] F. H. Yildiz and G. K. Schoolnik, "Role of rpoS in stress survival and virulence of Vibrio cholerae," Journal of Bacteriology, vol. 180, no. 4, pp. 773-784, 1998.
[19] S. P. Valeru, S. N. Wai, A. Saeed, G. Sandström, and H. Abd, "ToxR of Vibrio cholerae affects biofilm, rugosity and survival with Acanthamoeba castellanii," BMC Research Notes, vol. 5, article 33, 2012.

[20] S. Shanan, H. Abd, I. Hedenström, A. Saeed, and G. Sandström, "Detection of Vibrio cholerae and Acanthamoeba species from same natural water samples collected from different cholera endemic areas in Sudan," BMC Research Notes, vol. 4, article 109, 2011.

[21] H. Abd, T. Johansson, I. Golovliov, G. Sandström, and M. Forsman, "Survival and growth of Francisella tularensisin Acanthamoeba castellanii," Applied and Environmental Microbiology, vol. 69, no. 1, pp. 600-606, 2003.

[22] A. Saeed, H. Abd, B. Edvinsson, and G. Sandström, "Acanthamoeba castellanii an environmental host for Shigella dysenteriae and Shigella sonnei," Archives of Microbiology, vol. 191, no. 1, pp. 83-88, 2009.

[23] H. Abd, B. Wretlind, A. Saeed, E. Idsund, K. Hultenby, and G. Sandström, "Pseudomonas aeruginosa utilises its type III secretion system to kill the free-living amoeba Acanthamoeba castellanii," Journal of Eukaryotic Microbiology, vol. 55, no. 3, pp. 235-243, 2008.

[24] H. Abd, A. Weintraub, and G. Sandström, "Intracellular survival and replication of Vibrio cholerae $\mathrm{O} 139$ in aquatic free-living amoebae," Environmental Microbiology, vol. 7, no. 7, pp. 10031008, 2005.

[25] H. Abd, S. Shanan, A. Saeed, and G. Sandström, "Survival of Vibrio cholerae inside Acanthamoeba and detection of both microorganisms from natural water samples may point out the amoeba as a protozoal host for V. cholerae," Journal of Bacteriology and Parasitology, 2012.

[26] H. Abd, S. P. Valeru, S. M. Sami, A. Saeed, S. Raychaudhuri, and G. Sandström, "Interaction between Vibrio mimicus and Acanthamoeba castellanii," Environmental Microbiology Reports, vol. 2, no. 1, pp. 166-171, 2010.

[27] S. G. J. Smith, V. Mahon, M. A. Lambert, and R. P. Fagan, "A molecular swiss army knife: OmpA structure, function and expression," FEMS Microbiology Letters, vol. 273, no. 1, pp. 1-11, 2007.

[28] L. Zhou, R. Srisatjaluk, D. E. Justus, and R. J. Doyle, "On the origin of membrane vesicles in gram-negative bacteria," FEMS Microbiology Letters, vol. 163, no. 2, pp. 223-228, 1998.

[29] I. R. Henderson, F. Navarro-Garcia, M. Desvaux, R. C. Fernandez, and D. Ala'Aldeen, "Type V protein secretion pathway: the autotransporter story," Microbiology and Molecular Biology Reviews, vol. 68, no. 4, pp. 692-744, 2004.

[30] H. Kobayashi, K. Uematsu, H. Hirayama, and K. Horikoshi, "Novel toluene elimination system in a toluene-tolerant microorganism," Journal of Bacteriology, vol. 182, no. 22, pp. 6451-6455, 2000.

[31] M. R. Loeb, "Bacteriophage T4 mediated release of envelope components from Escherichia coli," Journal of Virology, vol. 13, no. 3, pp. 631-641, 1974.

[32] M. R. Loeb and J. Kilner, "Release of a special fraction of the outer membrane from both growing and phage T4-infected Escherichia coli B," Biochimica et Biophysica Acta, vol. 514, no. 1, pp. 117-127, 1978. 

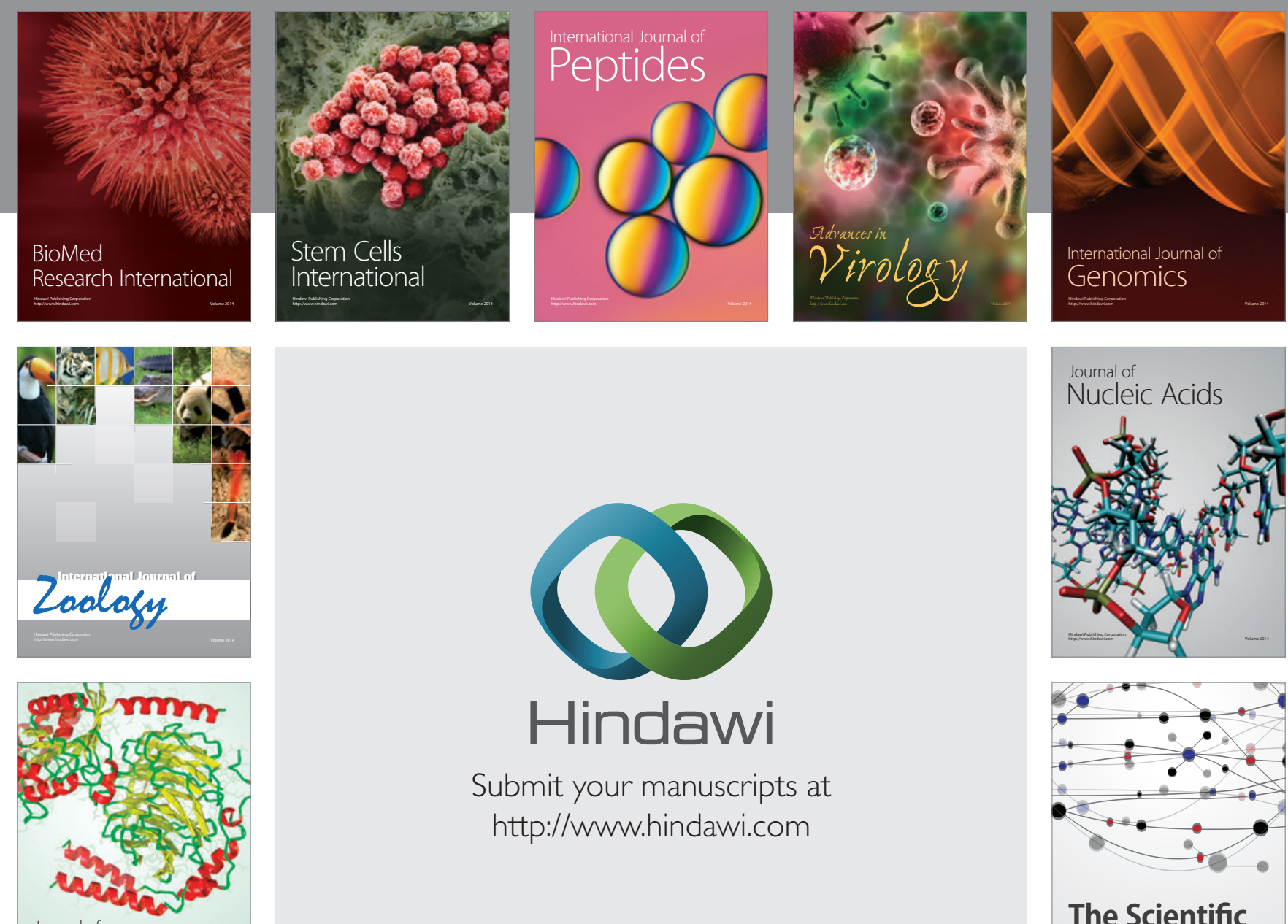

Submit your manuscripts at

http://www.hindawi.com

Journal of
Signal Transduction


The Scientific World Journal
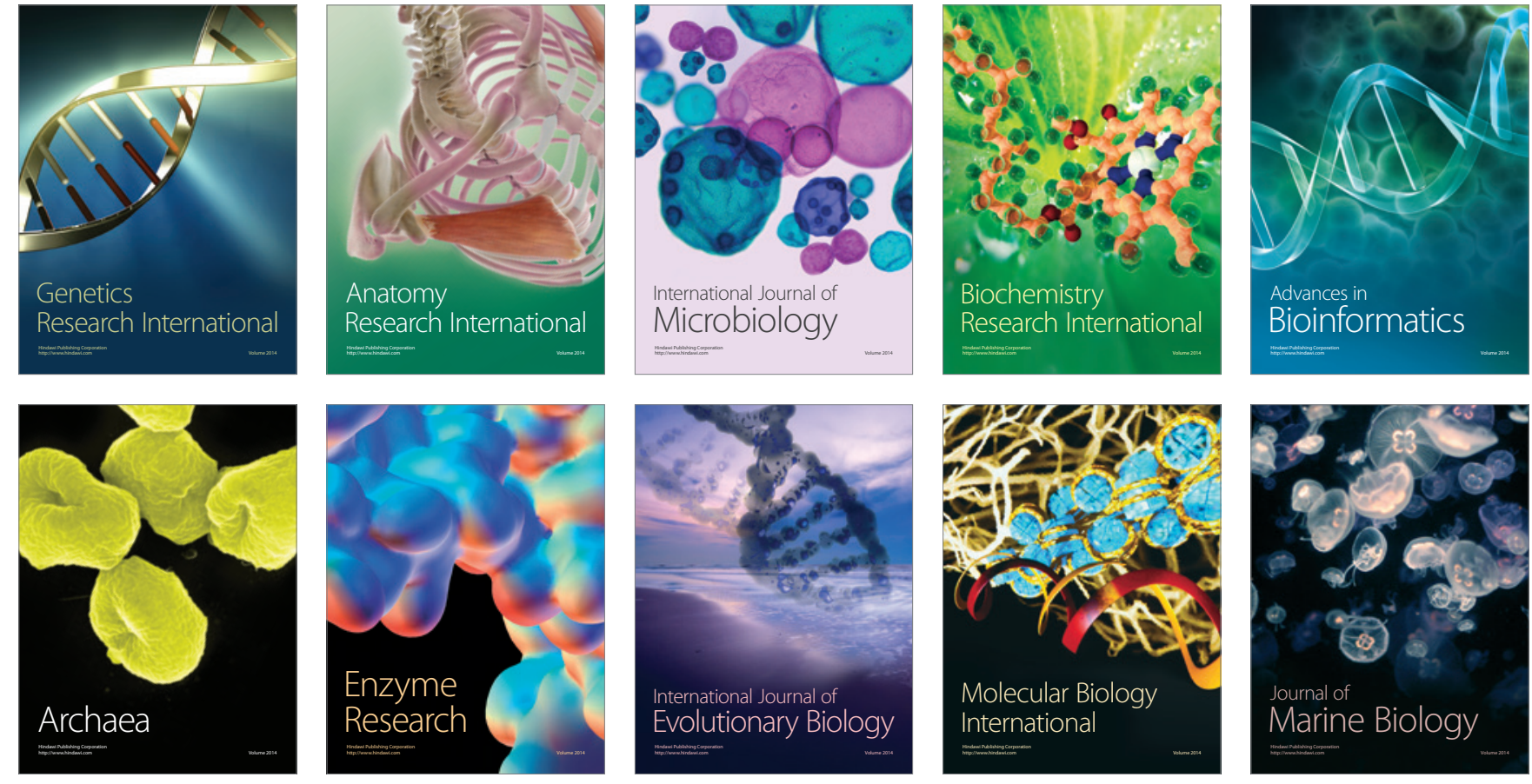